\title{
Studies on early association of Salmonella typhimurium with intestinal mucosa in vivo and in vitro: relationship to virulence
}

\author{
K. J. WORTON, D. C. A. CANDY*, T. S. WALLIS, G. J. CLARKE, M. P. OSBORNEt, S. J. \\ HADDON† and J. STEPHEN
}

Departments of Microbiology and †Physiology, and "Institute of Child Health, University of Birmingham, PO Box 363, Birmingham B15 2TT

\begin{abstract}
Summary. The abilities of six strains of Salmonella typhimurium to associate with rabbit ileal mucosa have been measured in vitro. Two were "virulent" strains (TML and W118 which are invasive and inducers of fluid secretion in rabbit ileal loops); four were "avirulent" (LT7, M206 and SL1027 which are invasive but induce negligible fluid secretion, and Thax-1 which is neither invasive nor an inducer of fluid secretion). A special organ-culture apparatus was designed to expose only the luminal surface of the mucosa to organisms. Viable counts of washed homogenised tissue taken 30 min after challenge showed that virulent strains TML and W118 and avirulent strains LT7 and M206 could not be distinguished from each other. Avirulent strain SL1027 associated less well than the other four strains, and Thax-1 associated less well than SL1027; both these strains were non-motile whereas the other four were motile. Thus, early association with gut mucosa did not discriminate all avirulent strains from the virulent strains. Qualitative examination of tissues by scanning electronmicroscopy did not detect strains LT7 and M206 on the mucosal surface whereas strains TML and W118 were readily seen, suggesting that the nature of association of virulent and avirulent strains was different. Qualitative examination by transmission electronmicroscopy of tissues challenged in vivo for 120 min showed virulent strains TML and W118 invading epithelial cells; similar events were reproduced after 120 -min challenge in vitro. In contrast, invasion by avirulent strains was observed only very rarely.
\end{abstract}

\section{Introduction}

The ability of a given strain of Salmonella typhimurium to cause secretion of fluid into ligated rabbit ileal loops (RILs) reflects its ability to cause diarrhoea in rhesus monkeys (Giannella et al., 1973) and, by inference, in man. Strains of salmonellae have been divided into three biotypes (Clarke et al., 1988): biotype $(++)$ which invades the ileal mucosa and causes fluid secretion, biotype $(+-)$ which invades the mucosa but induces negligible fluid secretion, biotype $(--)$ which neither invades nor causes fluid secretion.

The property of enterotoxin production in vitro does not per se account for the ability to induce fluid secretion (Wallis et al., 1986a). Moreover, invasion

Received 7 Dec. 1988; accepted 3 March 1989.

Correspondence should be sent to Dr J. Stephen, Department of Microbiology, University of Birmingham, PO Box 363, Birmingham B15 2TT. of mucosa, though an apparent pre-requisite for fluid secretion, does not per se result in secretion (Giannella et al., 1973; Wallis et al., 1986b). In this context, invasiveness has hitherto been defined in terms of the ability of organisms to be recovered from internal organs and blood some $18-24 \mathrm{~h}$ after intraluminal inoculation. However, degrees of invasiveness in the early stages of infection of intestinal mucosa have never been quantified.

This work was designed to see if one could discriminate the degrees of invasiveness in $(++)$ and $(+-)$ strains by quantitative assessment of the early stages of association of different strains with intestinal mucosa. The experiments were performed in vitro with a purpose-built system which allowed organisms to be introduced solely to the mucosal surface, thus simulating the conditions in vivo in which the only route of entry into the tissue is via the mucosal epithelium. The in-vitro approach was used because several strains could be compared 
in tissue from one animal, thereby eliminating potential inter-animal variations.

Conventional fragment organ cultures of intestinal mucosa were used initially because of their success with non-invasive gut pathogens, e.g., enteropathogenic Escherichia coli (Knutton et al., 1987). However, they were discarded when preliminary work showed that S. typhimurium penetrated into deeper tissue layers via the cut faces of the tissue. Qualitative electronmicroscopic studies were also made of mucosal invasion of tissue in the organ culture system, and these were compared.with morphological alterations produced by organisms invading similar mucosa in vivo.

\section{Materials and methods}

\section{Strains of S. typhimurium}

Six strains of $S$. typhimurium were studied: TML, W118, M206, SL1027, LT7, Thax-1. On the basis of their behaviour in the ligated rabbit ileal loop test (RILT), they are described as: $(++)$, invasive and causing fluid secretion (TML and W118); (+-), invasive but causing negligible fluid secretion (SL1027, LT7 and M206); and $(--)$, non-invasive and not causing fluid secretion (Thax-1). As indicated previously (Wallis et al., 1986a; Clarke et al., 1988), in the context of this work, only biotype $(++)$ is regarded as virulent.

For studies of invasion in vivo, only TML $(++)$, LT7 and SL1027 (+ - ) were used; the selection was based on data from in-vitro association studies (see Results). For other experiments, all strains were studied.

\section{Tissue culture media}

Two media were combined for maintaining organ cultures: Dulbecco's modification of Minimum Essential Medium (DMEM) containing sodium bicarbonate $2.25 \mathrm{~g} / \mathrm{L}$ and L-glutamine $584 \mathrm{mg} / \mathrm{L}$ (Flow Labs, Rickmansworth); and National Collection of Type Cultures (NCTC) medium 135 containing sodium bicarbonate $3 \cdot 70 \mathrm{~g} / \mathrm{L}$ (Flow Labs).

\section{In-vitro organ culture apparatus}

The organ-culture apparatus was constructed in the Medical Physics Department of Birmingham Maternity Hospital (see fig. 1). Equal volumes $(c .3 \mathrm{ml})$ of tissue culture medium (mixture of equal volumes of DMEM and NCTC 135) were added to each side of the tissue which was gassed continuously with $\mathrm{O}_{2} 95 \%, \mathrm{CO}_{2} 5 \%$ (British Oxygen Company, London). The whole apparatus fitted inside a $37^{\circ} \mathrm{C}$ incubator whilst in use. The tubes supplying gas entered the incubator through the hole in the roof which normally held a thermometer.

\section{Preparation of challenge inocula}

In-vitro mucosal association studies. Strains were stored short-term at $-20^{\circ} \mathrm{C}$ as a suspension in equal volumes of Mueller Hinton Broth (Oxoid) and glycerol. They were thawed to room temperature, plated on MacConkey Agar and incubated for $18-24 \mathrm{~h}$ at $37^{\circ} \mathrm{C}$. Approximately 50 colonies were picked from the plate, suspended in $10 \mathrm{ml}$ of Brain Heart Infusion Broth (BHI; Oxoid) and incubated for $2 \mathrm{~h}$ at $37^{\circ} \mathrm{C}$. Organisms were sedimented by centrifugation $(3500 \mathrm{~g}$, room temperature, $10 \mathrm{~min}$ ), washed once in $10 \mathrm{ml}$ of sterile saline $0.9 \%$ and resuspended in the tissue culture medium used in the organ-culture system, such that the total count was between $1 \times 10^{9}$ and $1 \times 10^{10}$ organisms $/ \mathrm{ml}$ as determined in a Helber counting chamber. This corresponded to a viable challenge of $\left(1 \times 10^{8}\right)-\left(1 \times 10^{9}\right) \mathrm{cfu} / \mathrm{ml}$.

RILT studies. Strains were grown on MacConkey Agar as above. Thereafter, the procedures were as described by Wallis et al. (1986a) with the following modification. Hartley Digest Broth (HDB), $80 \mathrm{ml}$, was inoculated with c. 50 colonies and incubated for $2 \mathrm{~h}$ with shaking at $37^{\circ} \mathrm{C}$. Organisms were then sedimented by centrifugation $\left(18000 \mathrm{~g}, 25 \mathrm{~min}, 4^{\circ} \mathrm{C}\right)$, resuspended in $2 \mathrm{ml}$ of fresh HDB, and samples were taken for viable count; $1 \times$ $10^{10} \mathrm{cfu} / \mathrm{ml}$ were introduced into 3-cm RILs, compared with $1 \times 10^{8} \mathrm{cfu} / \mathrm{ml}$ into 5 -cm loops (Wallis et al., 1986a). This increased, by $1.67 \times 10^{2}$, the number of inoculated organisms per $\mathrm{cm}$ of loop and helped to detect adherent organisms after relatively short incubation periods $(60-$ $120 \mathrm{~min}$, see below.).

\section{Total and viable counts}

Total counts were performed on organisms killed by formal saline. ( $\mathrm{HCHO} 10 \%$ in saline), in a Helber chamber. Viable counts were performed by the drop method of Miles et al. (1938).

\section{Animals}

Male Californian or New Zealand White rabbits (2.5$3.0 \mathrm{~kg}$ ) were used to obtain intestine for in-vitro work; preliminary experiments showed that similar results were obtained with either breed. Adult male New Zealand White rabbits of the same weight were used for in-vivo experiments.

\section{Quantitation of association: fixed 30-min incubation}

Bacterial inocula were prepared as previously described. Adult male rabbits were fasted overnight, anaesthetised with sodium pentobarbitone, and c. 20$30 \mathrm{~cm}$ of distal ileum was removed (proximal to the appendix); the animal was then killed by anaesthetic overdose. The tissue was opened longitudinally, and immediately placed in ice-cold pre-oxygenated tissueculture medium. Serosal muscle layers were removed by blunt dissection from 2-4-cm segments of ileum. The mucosal sheets were clamped between the two half- 
chambers, and bathed as above. Bacterial suspension ( $1 \mathrm{ml}$ in the same medium) replaced $1 \mathrm{ml}$ of medium on the mucosal side of the tissue.

After 30 min, medium was removed by aspiration, and the mucosa was detached and washed three times with $10 \mathrm{ml}$ of sterile saline $0.9 \%$. Tissue from one chamber was fixed for either light or electron microscopic examination; tissue from a similarly inoculated chamber was blotted dry, weighed (c. 8-20 mg) and homogenised on ice in a measured volume of sterile saline in a Sorval Omnimixer (Omni Corporation International, Wakerburg, CN, USA) at maximum speed for $30 \mathrm{~s}$. Viable counts were estimated on the homogenates and the results expressed as cfu/g of wet weight of mucosa.

Each strain was tested in at least five different experiments. Every experiment included two uninfected control chambers and two containing the $(++)$ strain TML as positive control because preliminary experiments showed that it associated well with ileal mucosa. In some experiments, an additional TML chamber was used for viable counts and compared with the "positive control" TML chamber; this gave an estimate of the reproducibility of the technique with respect to tissue from one animal, and the quantitative data were recorded separately (see fig. 6).

\section{In-vivo invasion in RILTs}

The RILT was used to compare in-vitro with in-vivo ultrastructural data (Wallis et al., 1986b). Relatively short incubation times of 60 and $120 \mathrm{~min}$ were chosen to establish the nature of association in the initial period of interaction. The construction of loops and subsequent analyses were as described by Wallis et al. (1986b).

\section{In-vitro invasion}

The organ-culture system was inoculated with a bacterial suspension as described above; incubation was at $37^{\circ} \mathrm{C}$ for 60 or $120 \mathrm{~min}$, followed by washing of the tissue three times in sterile saline, fixation and subsequent processing for electronmicroscopy.

\section{Electronmicroscopy}

Scanning electronmicroscopy (SEM). Tissues were immersed for $1 \mathrm{~h}$ in glutaraldehyde $2.5 \% \mathrm{w} / \mathrm{v}$ in $0.05 \mathrm{M}$ phosphate buffer, $\mathrm{pH} 7 \cdot 2$, total molarity adjusted with sucrose to $0.35 \mathrm{M}$. Acetone of graded concentrations $(70 \%, 90 \%$ and $100 \%)$ was used to dehydrate the specimens before critical point drying with liquid $\mathrm{CO}_{2}$ transition fluid, after which the tissue was fixed to copper stubs with electrically conductive paint (RS 555-156), sputtercoated with platinum, and examined in a JEOL $120 \mathrm{CX}$ II electronmicroscope fitted with a TEMSCAN and lanthanum hexaboride $\left(\mathrm{LaB}_{6}\right)$ filament. Images were obtained via a secondary electron detector with an accelerating voltage of $40 \mathrm{kV}$ and beam current of 10 $15 \mu \mathrm{A}$. Tissues were not post-fixed in $\mathrm{OsO}_{4}$ because
Osborne et al. (1984) had shown previously that this treatment destroyed extracellular membrane components such as cross-links between stereovilli in the mammalian cochlea.

Transmission electronmicroscopy (TEM) was as described by Wallis et al. (1986b).

\section{Detection of organisms}

No attempts were made to confirm that the organisms seen in either mode of electronmicroscopy were derived from the challenge inoculum. However, on the basis of the very low frequency of detection of pre-existing commensals in control preparations, we are confident that with strains TML and W118, the organisms seen were TML or W118; and we assume that the organisms seen when other strains were used were of experimental origin.

\section{Motility studies}

Wet preparation examination. Colonies were picked from MacConkey plates, inoculated into $\mathrm{BHI}$ broth, and incubated for $6 \mathrm{~h}$ and $24 \mathrm{~h}$ at $37^{\circ} \mathrm{C}$. A small sample of each was examined for motility in a Helber chamber by light microscopy.

Stab-culture in semi-solid agar. The semi-solid agar test described by Cruickshank et al. (1975) was used: organisms that produced a zone of growth with a distinct boundary close to the stab-line were considered nonmotile; those that produced a diffuse zone were considered motile.

\section{Flagella}

Organisms were examined for flagella by TEM after negative staining with phosphotungstic acid. Bacteria were grown in liquid and on solid media.

\section{Statistical analysis}

Students t-test. Viable counts (cfu/g) of the mucosal association achieved by the various strains in $30 \mathrm{~min}$ were compared with TML (positive control) by Students $t$-test after normalisation of the data by log transformation.

\section{Results}

\section{Motility}

By both direct microscopy and agar-diffusion, strains TML, W118, LT7 and M206 were motile; strains SL1027 and Thax-1 were non-motile (fig. 6). Strain SL1027 possessed flagella, but Thax-1 did not; flagella were readily seen also on all the motile strains. 


\section{Morphological preservation}

To validate the use of the apparatus (fig. 1), it was important to determine the time for which tissue survived, as this. would limit the duration of experiments. As judged by light microscopy, there was slight variation between chambers in the same experiment, but usually tissue was well preserved up to $30 \mathrm{~min}$; and this was chosen as the incubation period because we wished to assess the very early stages of bacterial association with host cells.

After incubation for $30 \mathrm{~min}$, SEM showed uninfected and infected tissues to be morphologically indistinguishable. Villi were tongue- and leafshaped, characteristic of rabbit ileum. Both infected and non-infected tissue showed some surface wrinkling. However, gross architectural integrity of villi and microvilli was good (figs. 2 and 3). Occasionally in control tissue, some elongation and budding of microvilli of individual cells was evident, probably due to senescent cells being extruded. This type of brush border disruption was, however, seen much more commonly in tissue infected with strains TML and W118. After 120 min, the tissue preservation varied from very good, with intact whole villi visible (fig. 4), to less good,

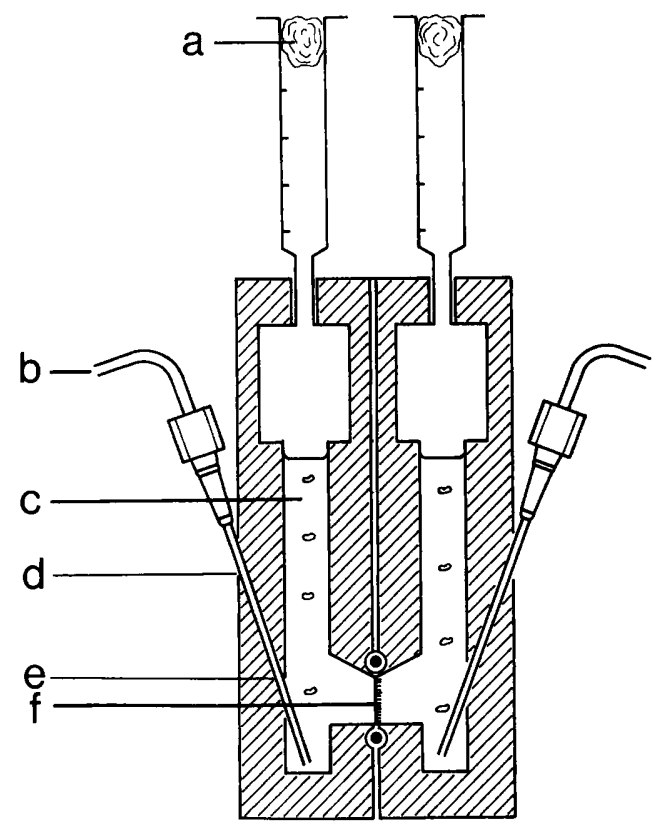

Fig. 1. A transverse section of the organ culture apparatus, consisting of two halves each containing eight chambers which pair on assembly; they are separated at the bottom by tissue clamped in situ between rubber O-rings. (a) Bubble trap seal; (b) oxygen inlet; (c) oxygenated tissue culture medium; (d) point of entry of needle into perspex; (e) point of exit of needle from perspex; and (f) mucosa. with some villus atrophy and enterocyte shedding (fig. 5).

These results demonstrate that tissue incubated for $30 \mathrm{~min}$ was suitable for quantitative assessment of organism-tissue association in this organ culture system. Mucosae incubated for $60-120 \mathrm{~min}$ were used for electronmicroscopic observations of selected areas where the general morphological appearance was good.

\section{Quantitation of bacterial association}

Bacterial association with rabbit ileal mucosa after $30 \mathrm{~min}$ is summarised in fig. 6. In each of 17 experiments, three strains were tested in duplicate chambers; and from a challenge inoculum of $c .1 \times$ $10^{8} \mathrm{cfu} / \mathrm{ml}$, only $1 \%$ was recovered from the tissue after $30 \mathrm{~min}$.

Strains SL1027 and Thax-1 associated significantly less well than strain TML ( $p$ [SL1027] $<0.02$; and $\mathrm{p}$ [Thax -1$]<0.001)$. The other strains showed no significant difference in their ability to associate with ileal mucosa. The data indicate that whereas avirulent strains LT7 and M206 could not be distinguished from virulent strains TML and W118, avirulent strains SL1027 and Thax-1 could be distinguished.

Morphological studies on the initial association of $S$. typhimurium with rabbit ileal mucosa infected in vivo

SEM with strain $T M L$. Generally the effects observed were similar to those previously demonstrated in our laboratory with the same in-vivo model, at this and at later time-points in infection (Wallis et al., 1986b). TML organisms could be found relatively frequently in microcolonies associating both with residual mucus, not removed during preparation for microscopy, and with ileal brush border (fig. 7). Bacteria were seen bound together by filamentous material; some projections on to the mucosal surface appeared to emanate from the bacterial surface. Bacteria were also seen intimately associated with elongations of brush border microvilli. These seemed to be induced by organisms in contact with mucosal surfaces (fig. 8); the surrounding brush borders of adjacent cells appeared relatively normal.

SEM with strains LT7 and SL1027. These strains were not seen in great numbers in association with either mucus or the brush border. Fig. 9 illustrates the characteristic appearance of the association of these strains with the mucosa: there was no brush border disruption either in the vicinity of, or at the point of contact between, bacteria and enterocytes; 


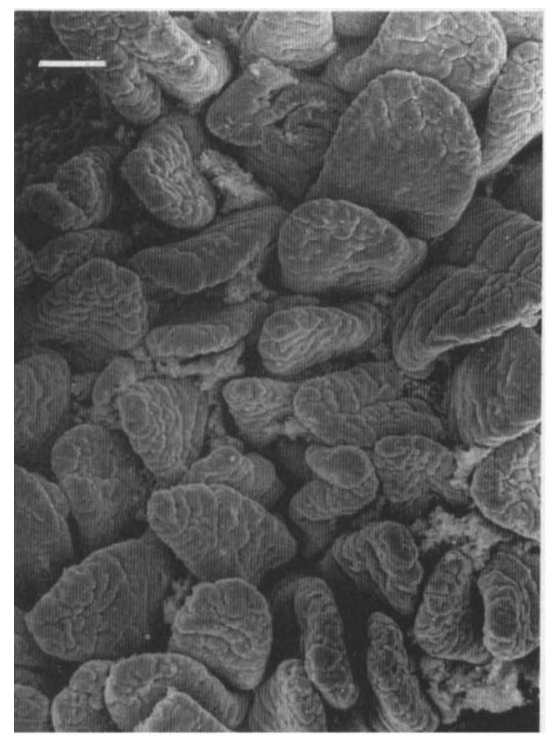

Fig. 2. Infected tissue after incubation for $30 \mathrm{~min}$, showing gross architecture. Note the tongue- and leaf-shaped villi characteristic of rabbit ileum. Gross architectural integrity was good in both uninfected and infected tissue which at this stage were indistinguishable; so only one micrograph is shown. SEM Size bar $=150 \mu \mathrm{m}$.

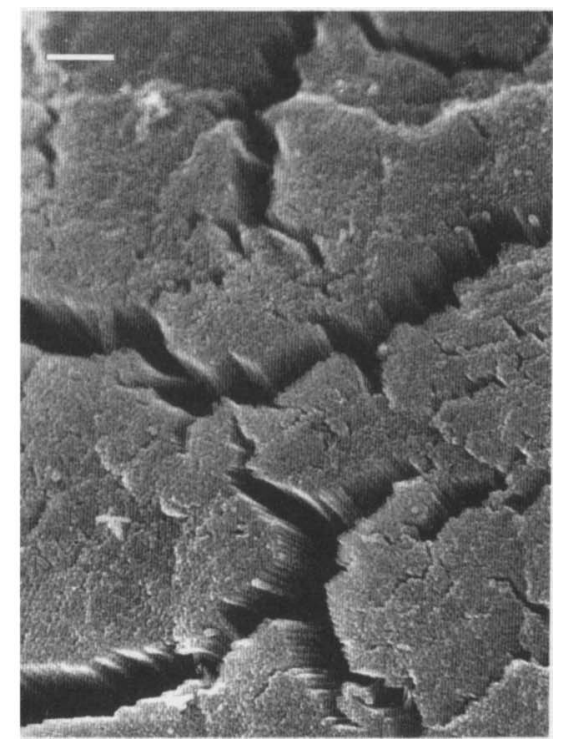

Fig. 3. Uninfected tissue after incubation for $30 \mathrm{~min}$, showing microvilli. The enterocyte brush border is intact although some separation of the microvilli indicates either degenerative changes or possibly preparation artifacts. SEM Size bar $=1 \cdot 3 \mu \mathrm{m}$.
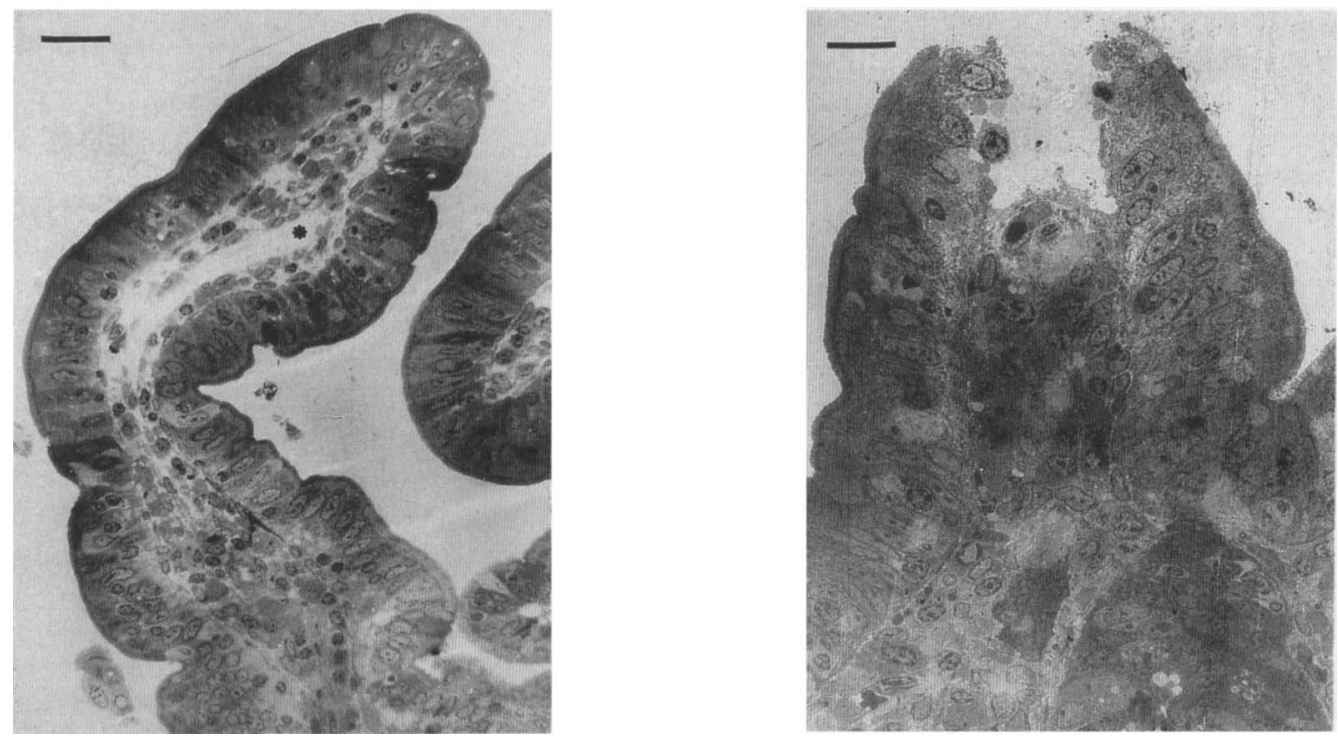

Figs. 4 (left) and 5 (right). Uninfected tissue after incubation for $120 \mathrm{~min}$. The epithelium was either intact (fig. 4), or less well preserved, showing villus atrophy and loss of enterocytes (fig. 5). Note the slight oedema $\left(^{*}\right)$ in the upper villus region in fig. 4 . TEM Size bar $=25 \mu \mathrm{m}$ (fig. 4); $13 \mu \mathrm{m}$ (fig. 5).

but there were structures linking the organisms and host which could be of either bacterial or host origin.

TEM with strain TML. Induced morphological alterations were similar to those previously dem- onstrated in our laboratory with the same in-vivo model, at this and at much later time-points in infection (Wallis et al., 1986b). However, they were never seen in tissue infected with either of the avirulent strains, SL1027 and LT7. Fig. 10 shows 


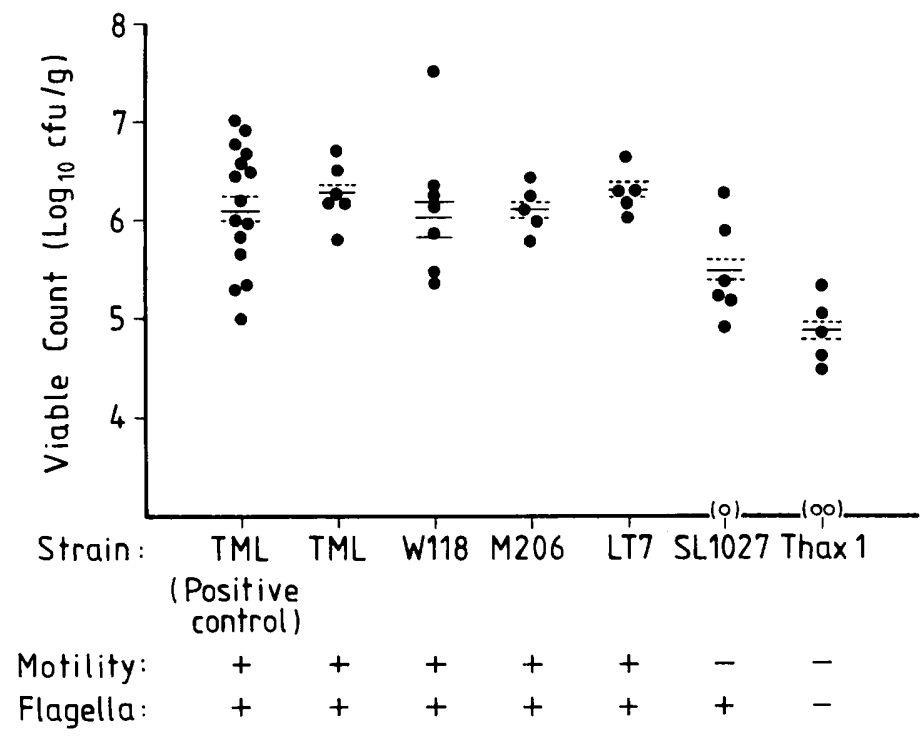

Fig. 6. Association of bacterial strains with ileal mucosa after incubation for $30 \mathrm{~min}$. Each point (O) represents a separate experiment to estimate the viable bacteria associated with mucosal cells. Mean association values for strains SL 1027 and Thax-1 were significantly different from those of strains TML, W118, LT7 and M206 (see text). There was no significant difference between the viable counts from TML chambers and the positive control chambers on the occasions when these were compared:- - mean values; - - standard errors; $O$ experiments in which no viable bacteria were recovered.

early stages of invasion by bacteria within an enterocytic vacuole: elongation of the microvilli is evident; mitochondria in the apical cytoplasm were undisturbed; contained within the vacuole were microvillus fragments; bacterial cytoplasm exhibited electron-lucent areas. Although bacteria were invading host cytoplasm in the close vicinity of tight junction complexes, the latter remained intact.

\section{Morphological studies on the initial association of $S$. typhimurium with rabbit ileal mucosa infected in vitro}

SEM with strain TML. After incubation for 60 min, it was relatively easy to find organisms attached to the brush border, which was disrupted at the point of contact between bacteria and microvilli; filamentous structures, which could be of either host or bacterial origin, were also observed connecting the bacterial surface to the mucosa (fig. 11). This was preceded by a sequence of events similar to that described below for strain W118 (figs. 12 and 13).

$S E M$ with strain $W 118$. Again, it was relatively easy to find bacteria associated with the mucosa (fig. 12) and embedded to varying degrees within mucus layers covering parts of the brush border. There was obvious brush-border disruption in the vicinity of, and at the point of contact between, organisms and host cells and clear evidence of bridging structures linking bacteria and host cells (fig. 13). In fig. 14, numerous bacteria are intimately associated with a mucosal cell whose microvilli are elongated; adherent bacteria appear to be showing penetration.

SEM with strains M206, LT7, SL1027 and Thax1. In contrast, strains M206, LT7, SL1027 and Thax-1 were difficult to find at any time in association with the mucosa, and were never seen in microcolonies as large as those seen with strains TML and W118. However, although strain LT7 was shown to be attached via filamentous structures to brush border, this was not accompanied by any obvious microvillus elongation (fig. 15, 15a); no extensive association with overlying mucus was seen. Strain SL1027 was difficult to find, and strain Thax-1 very rarely seen. As stated in Methods, the organisms found in such small numbers on tissue infected with the above strains were assumed, but not proven, to be $S$. typhimurium.

$T E M$ demonstrated bacteria invading ileal mucosa after infection of tissue in the organ culture system (fig. 16, strain TML; fig. 17, strain W118). It was relatively easy to find multiple organisms in the early stages of invasion, and host tissue which demonstrated the principal morphological features of invasion (seen in vivo and described above) including elongation, budding and vesiculation of the brush border; nearby mitochondria appeared 


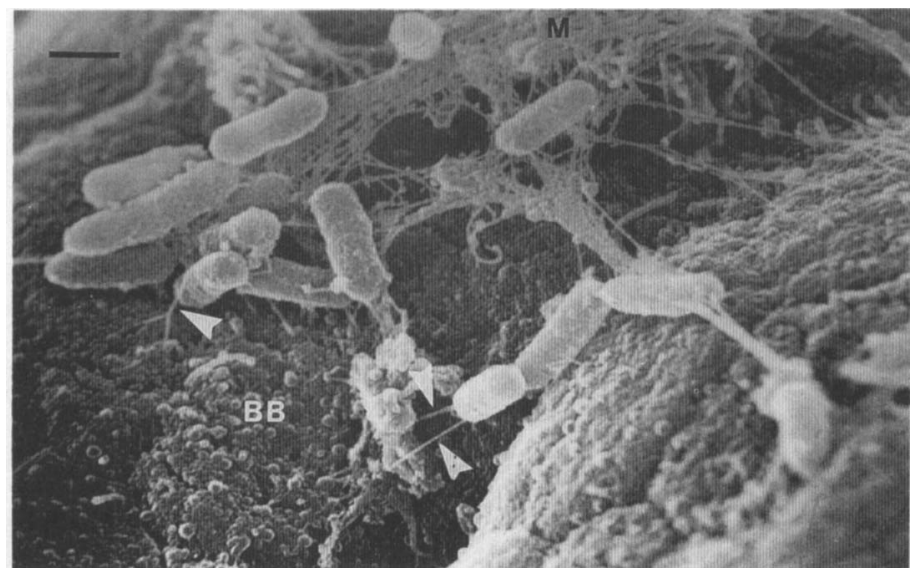

Fig. 7. Association of strain TML with mucosa after incubation for $120 \mathrm{~min}$ in vivo showing bacteria associated with mucus (M) and with the brush border (BB). Note the filamentous structures connecting bacteria to the mucosal surface (white arrow heads). SEM Size bar $=1 \mu \mathrm{m}$.

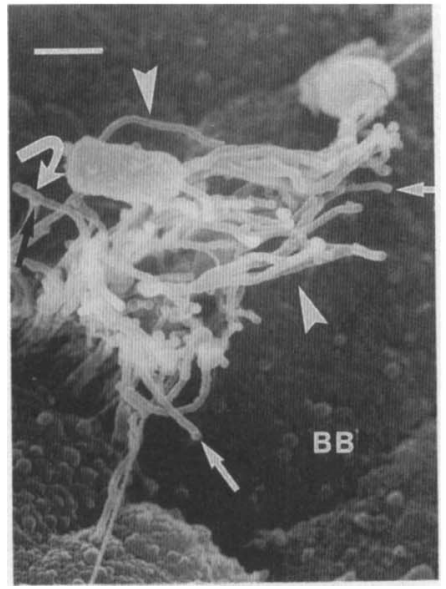

Fig. 8. Same as fig. 7, showing microvilli grossly elongated (white arrow heads), swollen at the tips (straight arrows) and segmented (curved arrows) in the region of bacterial contact. The brush border (BB) of surrounding cells appears relatively normal morphologically, although incipient swelling of microvilli is present. SEM Size bar $=1.0 \mu \mathrm{m}$.

healthy. No invasion was seen via intercellular junctional complexes.

\section{Discussion}

We have studied, probably for the first time in a relevant model in vitro, the sequence of events surrounding the initial association with and invasion of intestinal mucosa by $S$. typhimurium. A special apparatus was developed to allow infection of epithelia from the luminal side only; but, like

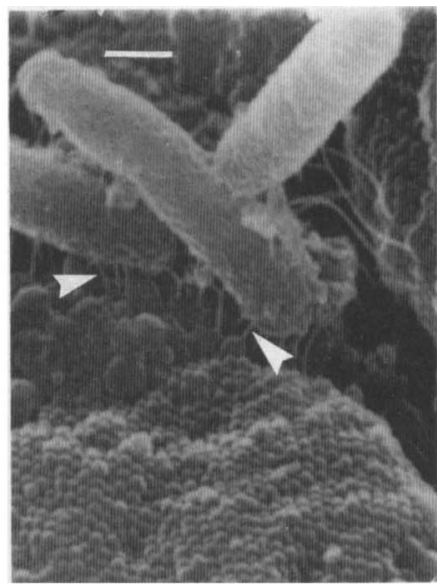

Fig. 9. Association of strain SL 1027 with mucosa after incubation for $120 \mathrm{~min}$ in vivo. This is representative of observations when $(+-)$ strains were used. Bacteria were attached to the brush border which appeared morphologically normal. Note the bridging filaments between the organisms and host tissue (arrow heads). SEM Size bar $=0.5 \mu \mathrm{m}$.

every model, it is not ideal. Its technical advantages and disadvantages are discussed together with data on the comparative behaviour of six strains of $S$. typhimurium, with different biological properties, in vitro and in vivo.

The main restriction of the model is the relatively short time $(2 \mathrm{~h})$ for which the tissue could be kept structurally intact; and this limited the period during which meaningful studies could be made on bacterial interactions. More effective oxygenation might prolong structural integrity; however, from 


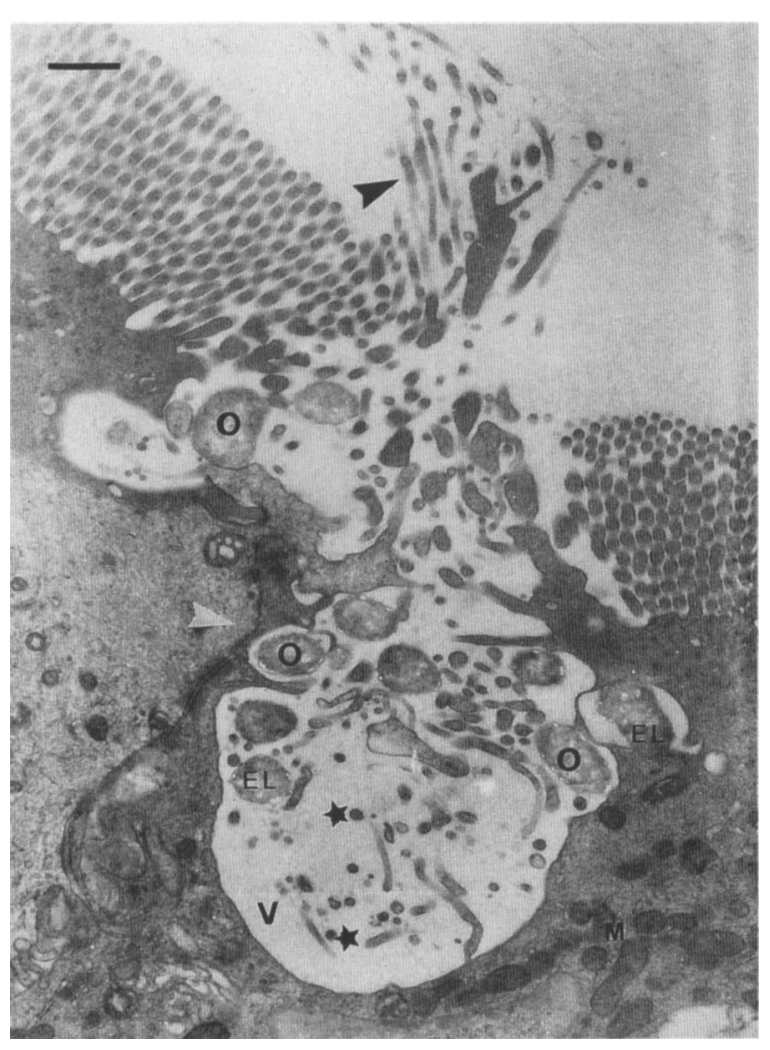

Fig. 10. Invasion of strain TML after incubation for $120 \mathrm{~min}$ in vivo: invasion by organisms ( 0 ); elongation of microvilli (black arrow head); intact apical cytoplasm near the point of contact, with morphologically normal mitochondria (M); organisms within vacuole $(\mathrm{V})$ containing microvillus fragments $\left({ }^{*}\right)$; no disruption of intercellular boundaries (white arrow head); electron-lucent (damaged?) organisms (EL). TEM Size bar $=0.45 \mu \mathrm{m}$.

unpublished studies within our group, on murine intestine, it is clear that even so, in the absence of a functioning blood supply and lymphatic drainage, intestinal tissue does not survive long in vitro. The continued inward transport of ions and fluid by enterocytes is associated with fluid accumulation in the lamina propria, detachment of the epithelium from the lamina propria and vacuolation of enterocytes. Tissue preservation is much better at $4^{\circ} \mathrm{C}$ than at $37^{\circ} \mathrm{C}$, presumably due to reduction in metabolic activity and rate of active inward transport of ions which is the driving force for fluid uptake. Thus, with present technology, study of association with and invasion of intestinal mucosa by bacteria in vitro must be restricted to the earliest events.

In our quantitative assessment of bacterial association with intestinal mucosa, achieved in
30 min, we compared four "avirulent" strainswhich caused negligible fluid secretion in the RILT (Wallis et al., 1986b)-with the "virulent" strain TML which did cause fluid secretion. In their association with mucosa (fig. 6), two avirulent strains (SL1027 and Thax-1) could be distinguished from strain TML; but the other two (LT7 and M206) could not. The inability of strains LT7 and M206 to cause fluid secretion, therefore, cannot be attributed to a decreased potential for initial mucosal association in comparison with virulent strains.

A factor which may be important in the pathogenicity of $S$. typhimurium is the possession of flagella. Motility could increase the number of interactions achieved by luminal bacteria with host mucosa, which might lead to adherence. Of our six strains, only SL1027 and Thax-1 were non-motile, and they showed significantly less association than strain TML after $30 \mathrm{~min}$. It is thus probable that motility contributes to initial association. It is unclear whether the flagella are merely organelles of motility which increase the frequency of contact, or whether they function as adhesins; and this requires further investigation. So, with the nonmotile but flagellate strain, SL1027, we cannot decide whether loss of motile function or structural change in the flagella is the cause of less initial association.

The significance of the quantitative data cannot be appreciated fully without reference to electronmicroscopy studies. SEM of infected mucosa showed that $(++)$ strains, which were both invasive and inducers of fluid secretion in the RILT (Wallis et al., 1986b), were consistently easier to find in varying degrees of association with mucosa than either $(+-)$ strains, which were invasive but induced negligible fluid secretion, or a $(--)$ strain, which was neither invasive nor an inducer of fluid secretion. Data on mucosal association suggested that strains LT7 and M206 should be found by electronmicroscopy as frequently as strains TML and W118; they were not. Two hypotheses might explain this apparent anomaly. First, strains LT7 and M206 may have a characteristically more diffuse type of adherence to mucosa so that, although the viable counts of homogenised tissue were the same, the bacteria were more difficult to find by electronmicroscopy. Second, strains LT7 and M206 may not associate as avidly or as specifically (involving adhesins?) as the $(++)$ strains: the preparative techniques for electronmicroscopy might remove bacteria from the mucosa more easily than the milder washing before homogenisation procedures for viable counts. 


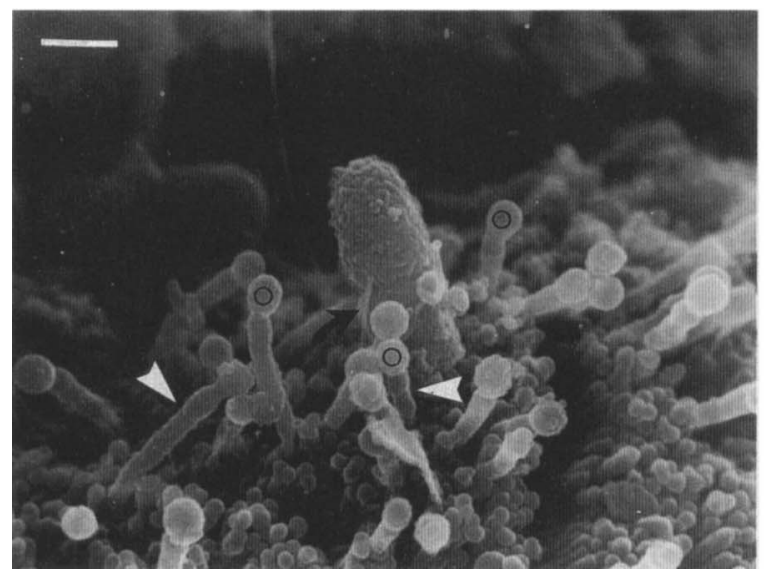

Fig. 11. Association of strain TML after incubation for $120 \mathrm{~min}$ in vitro. Filamentous structures connecting bacteria to brush border (curved arrow); elongated microvilli with swollen tips (black circles); and segmentation of elongated microvilli (white arrow heads). SEM Size bar $=0.5 \mu \mathrm{m}$.

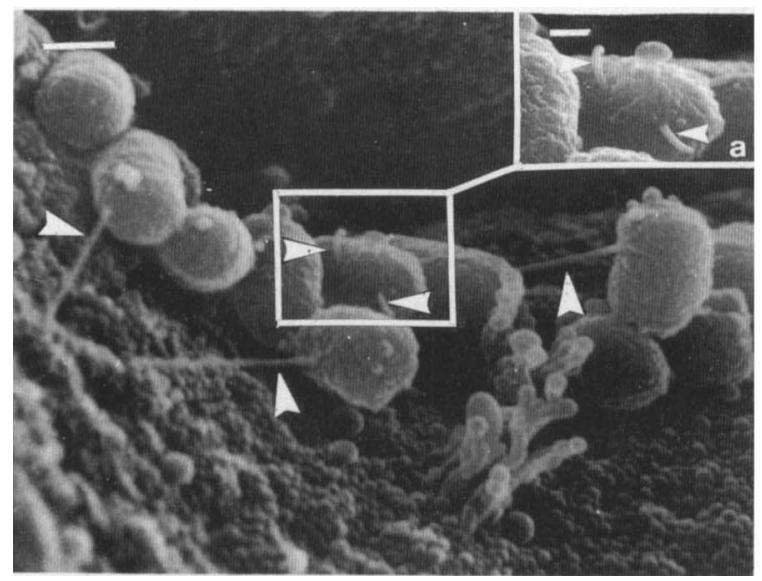

Fig. 13. Same as fig. 12, showing organisms anchored by bridging structures (white arrow heads) to the underlying brush border. Inset shows filamentous structures which are probably bacterial in origin; these have the same diameter as the complete ones (white arrow heads) and could be broken or developing linkages. Note also the elongation of microvilli (in the vicinity of the adherent organisms) with swollen tips and segmentation. SEM Size bar $=0.5 \mu \mathrm{m}$.

In the quantitative studies, with the exception of strains SL1027 and Thax-1, the recovery of organisms was only $c .1 \%$ of the challenge inoculum. The reason for the low numbers of organisms attaching could be that there was only a limited number of sites on mucosa susceptible to bacterial attachment, so that "saturation" was reached with

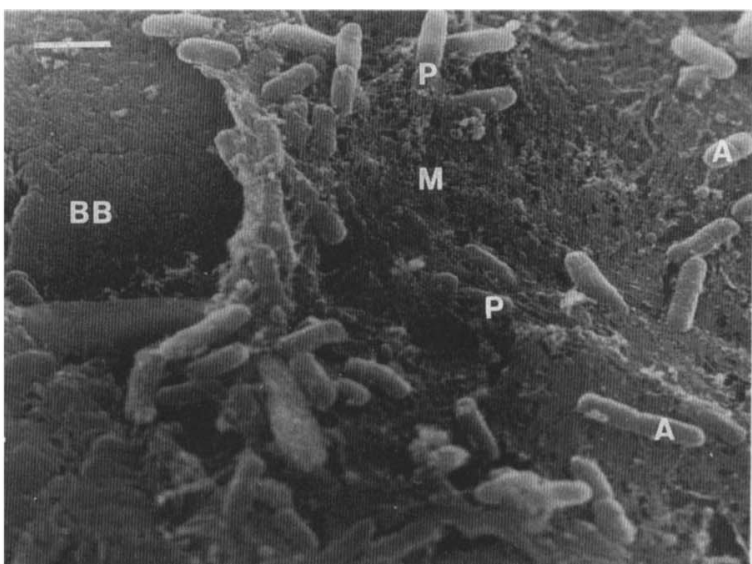

Fig. 12. Association of strain W118 after incubation for $120 \mathrm{~min}$ in vitro. This shows association with mucus $(M)$ overlaying brush border (BB). Individual organisms are in various stages of attachment (A) or penetration (P). SEM Size bar $=2 \cdot 3 \mu \mathrm{m}$.

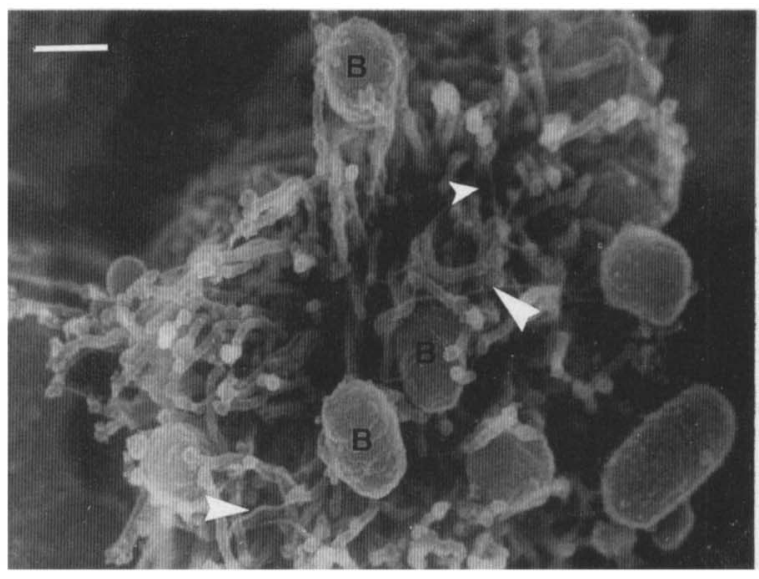

Fig. 14. Same as figs. 12 and 13, depicting a stage estimated to be later than that in fig. 13 with a group of organisms becoming internalised. The bridging structures are less clear than in fig. 13; but some thread-like filaments (white arrow heads) are evident, of the same diameter as those arrowed in fig. 13, but apparently detached from the bacterial surfaces and not under tension. Bacteria (B) are wrapped around with elongated microvilli. The cell depicted is separated from adjacent "normal" cells, possibly becoming detached. SEM Size bar $=0.7 \mu \mathrm{m}$.

relatively few organisms. This is unlikely because, in preliminary experiments (data not shown), a 10fold increase of the inoculum led to recovery of more organisms, but again only $1 \%$ of the inoculum; and this suggests that only a small fraction of the inoculum expressed the properties for successful association with the mucosa. If so, organisms 


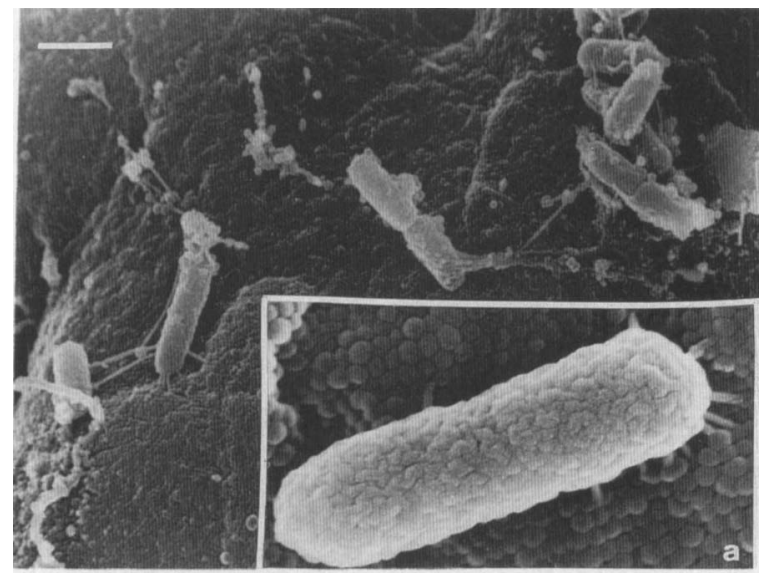

Figs. 15 and 15a. Association of strain LT7 after incubation for $60 \mathrm{~min}$ in vitro. The brush border appears normal; bridging structures again appear to anchor bacteria to the brush border, but without brush-border perturbation such as that induced by either TML or W118. Fig. 15a is a higher magnification of a separate field showing, more clearly, bridging structures between organisms and brush border. SEM Size bar $=1.5 \mu \mathrm{m}$ (fig. 15), $0.4 \mu \mathrm{m}$ (fig. 15a).

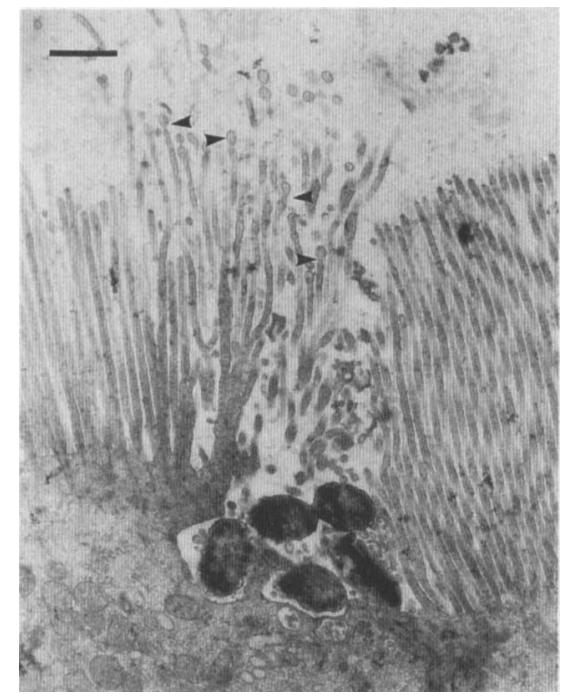

Fig. 16. Invasion of strain TML after incubation for $120 \mathrm{~min}$ in vitro. This shows brush-border disruption at the site of invasion: microvillus elongation, budding and segmentation at the tips (black arrow heads). The morphological changes are very similar to those seen in vivo. TEM Size bar $=0.9 \mu \mathrm{m}$.

isolated from colonised tissue ought to contain a higher percentage of the adherent phenotype. Attempts to show this, however, proved difficult without intermediate culture in laboratory media which defeated the object. An alternative reason for the low number of initially adherent organisms

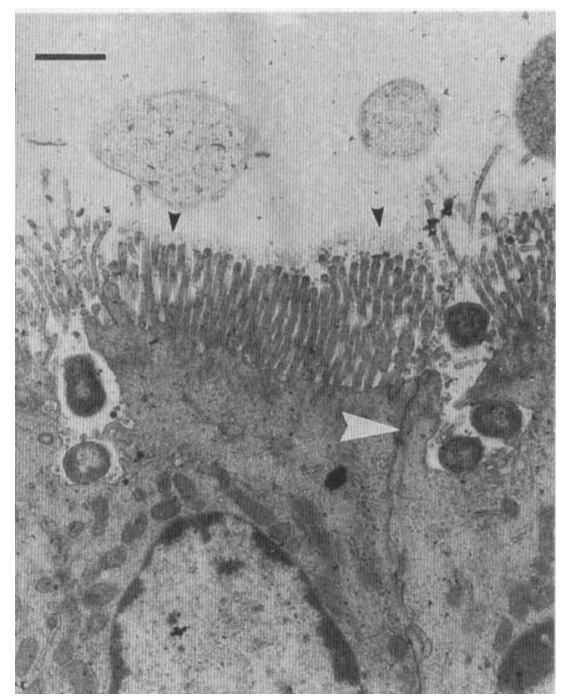

Fig. 17. Invasion of strain W118 after incubation for $120 \mathrm{~min}$ in vitro. This shows similar features to those in fig. 16. In addition, there is an example of bacterial penetration without disruption of tight junctions (white arrow head). The mitochondria in the apical cytoplasm appear healthy, and the glycocalyx overlaying the brush border between the two invasion sites seems intact (black arrow heads). TEM Size bar $=0.6 \mu \mathrm{m}$.

is that the time of incubation was too short. However, tissue degeneration was progressive beyond $30 \mathrm{~min}$, hence the biological significance of increased numbers of organisms recovered in tissue homogenates would be open to question.

Whereas quantitative studies of the initial association did not discriminate all avirulent strains from virulent strains, qualitative electronmicroscopy studies did: only for strains TML and W118 were attachment and invasion found with relatively high frequency.

SEM examination of organisms associated with mucosa revealed various structures which appeared to emanate from the surface of bacteria. These included long flagella-like processes which appeared to extend from bacteria on to the mucosa, and shorter appendages which may have been the remains of broken off flagella. Differentiation between bacterial and host origin of these structures may best be done by immuno-electronmicroscopy with appropriate antisera.

TEM was also used to study the phenomenon of invasion. As noted previously, complete villus architectural integrity was not maintained routinely beyond $30 \mathrm{~min}$. However, there were always sufficient areas of tissue which had not degenerated, or which had not been expelled from the tissue as a consequence of infection, in which meaningful 
observations of bacterial invasion could be made. We believe, therefore, that the qualitative statements derived from the work are biologically meaningful.

TEM showed that mucosal invasion in vitro generated the same morphological alterations as were observed in vivo (this work; Takeuchi, 1967; Takeuchi and Sprinz, 1967; Wallis et al., 1986b). However, we did not observe, as Takeuchi (1967) claimed, organisms penetrating the mucosa through intercellular junctional complexes. The observed features included localised brush-border disruption (microvillus elongation, "budding" and vesiculation) and progression of organisms into vacuoles within the apical cellular cytoplasm. These phenomena were observed only in areas where $(++)$ strains were associated with mucosa, and are therefore most likely to be bacterially induced, though the nature and mechanism of the bacterial determinants which trigger this host response remain to be determined.

There seems little doubt that the brush-border derangements and subsequent events are bacterially induced; but the precise point in the organismmucosa interaction at which the derangements are triggered is not clear. The derangement may result from initial attachment and a specific signalling event; e.g., SEM shows organisms attaching to microvilli which are elongated and swollen at the tips. However, although TEM micrographs confirm this elongation and swelling, invading bacteria were already intimately associated with the terminal web; and it was hard to find, by TEM, organisms actually attached to microvilli in the early stages of association. Therefore it is difficult to deny the possibility that the effects seen in SEM were produced by organisms already in association with the terminal web, which would not be visible in SEM. However, it is equally possible that failure to find, in TEM, organisms in the earliest stages of attachment could be due to sampling problems.

Conclusions. (1) Technically, we have succeeded to a limited degree in demonstrating, in vitro, the early events in $S$. typhimurium invasion. The limiting factor is the time (possibly not more than
$2 \mathrm{~h})$ for which tissue integrity can be maintained. (2) At least two kinds of very early association are exhibited: one involves a loose non-specific association, possibly with superficial mucus; the other involves attachment of organisms to brush borders of epithelial cells, which could be specific-adhesin mediated. This distinction could not have been made without the qualitative electronmicroscopy evidence complementing the quantitative association data. (3) Motility almost certainly plays some part in facilitating association. (4) The cellular origin, nature and role of the bacterial brush-border bridging structures have yet to be, and could well be, resolved by use of this system coupled with immuno-electronmicroscopy. (5) Association of virulent $S$. typhimurium strains is not a Vibrio cholerae-like or enterotoxigenic $E$. coli-like anchor attachment of organisms: $V$. cholerae and $E$. coli do not go on to invade the cells to which they adhere. It would rather appear that with $S$. typhimurium attachment is the first step in a biological continuum which leads to penetration of enterocytes with subsequent invasion into deeper tissues. The functional significance of attachment by virulent strains is almost certainly different from that of avirulent ones: maybe only with the former is a transmembrane signal transmitted to initiate the internalisation process. (6) Quantitation of invasion in this system would encounter the problem of tissue preservation beyond $2 \mathrm{~h}$; and this vital aspect of $S$. typhimurium infection may best (or only) be solved by using short length RILs for longer periods of time. However, varying kinds of response of rabbits were observed by Wallis et al. (1986b), such as variable shedding of epithelial cells containing bacteria, in acute infections; and this may explain the variability seen in association data (not shown) in our preliminary experiments in vivo.

D.C.A.C., M.P.O and J.S. gratefully acknowledge the financial support of the Wellcome Trust, and the Central Birmingham Health Authority Endowment Fund for support of K.J.W. We also thank Mr G. Hollins of the Medical Physics Department of the Queen Elizabeth Maternity Hospital for skilled technical assistance in the construction of the organculture apparatus.

\section{REFERENCES}

Clarke G J et al. 1988 Expression of an antigen in strains of Salmonella typhimurium which reacts with antibodies to cholera toxin. Journal of Medical Microbiology 25 : 139-146.

Cruickshank R, Duguid J P, Marmion B P, Swain R H A (eds) 1975 Tests for identification of bacteria. In: Medical microbiology: a guide to the laboratory diagnosis and

control of infection, 12 th edn, vol 2. The practice of medical microbiology. Churchill Livingstone, Edinburgh, pp 170189.

Giannella R A, Formal S B, Dammin G J, Collins H 1973 Pathogenesis of salmonellosis. Studies of fluid secretion, mucosal invasion and morphologic reaction in the rabbit ileum. Journal of Clinical Investigation 52: 441-453.

Knutton S, Lloyd D R, McNeish A S 1987 Adhesion of 
enteropathogenic Escherichia coli to human intestinal enterocytes and cultured human intestinal mucosa. Infection and Immunity 55: 69-77.

Miles A A, Misra S S, Irwin J O 1938 The estimation of the bactericidal power of the blood. Journal of Hygiene 38: 732749

Osborne M P, Comis S D, Pickles J O 1984 Morphology and cross-linkage of sterocilia in the guinea-pig labyrinth examined without the use of osmium as a fixative. Cell and Tissue Research 237: 43-48.

Takeuchi A 1967 Electron microscope studies of experimental Salmonella infection. I. Penetration into the intestinal epithelium by Salmonella typhimurium. American Journal of Pathology 50: 109--136.
Takeuchi A, Sprinz H 1967 Electron microscope studies of experimental salmonella infection in the preconditioned guinea pig. II. Response of the intestinal mucosa to invasion by Salmonella typhimurium. American Journal of Pathology 51: $137-147$.

Wallis T S, Starkey W G, Stephen J, Haddon S J, Osborne M P, Candy D C A 1986 a Enterotoxin production by Salmonella typhimurium strains of different virulence. Journal of Medical Microbiology 21 : 19-23.

Wallis T S, Starkey W G, Stephen J, Haddon S J, Osborne M P, Candy D C A $1986 b$ The nature and role of mucosal damage in relation to Salmonella typhimurium-induced fluid secretion in the rabbit ileum. Journal of Medical Microbiology 22 : 3949. 